\title{
Pwotéjé ak Plywood ${ }^{1}$
}

Hal S. Knowles, III, Kathleen C. Ruppert, Karla A. Lenfesty ak Barbara Haldeman²

Bon jan pwoteksyon pou siklòn mandé tan ak lajan. Lè pa genyen ase tan pou nou jwen yon kontraktè komèsyal ouswa pa genyen ase lajan, panno plywood ki byen enstalé kapab buy bon jan pwoteksyon pou kay-nou.

Paske panno plywood pa diré lontan, pwa panno-a, ak tan ke sap pran pou enstalé, nou rekòmandé ke nou pa itilizé-yo a mwen ke nou pa genyen lòt bagay. Pase pran dokiman nou-an ki réle "Enstalé Panno Siklòn Pou Fénèt” pou nou kapab étidyé lòt altènatif.

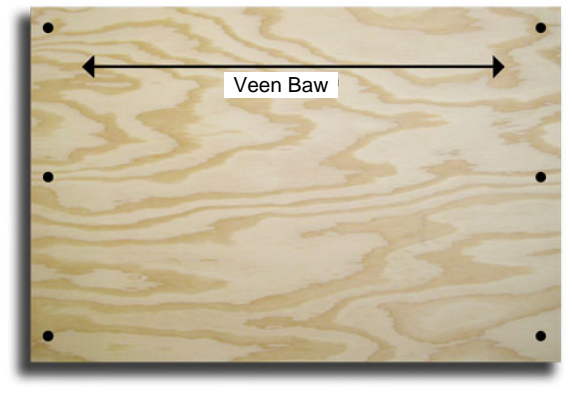

Plywood pi fò nan direksyon venn bwa-a. Sous: Enstiti pou Sekirité Biznis ak Kay

\section{Èske Tout Panno Plywood Se Menm?}

NON. Genyen diferan fason pou enstalé panno plywood. Sepandan, nou mandé ké ou itilizé youn dan dé gwo métòd-yo. Pou kay ki fèt ak blòk siman ak fénèt ki konstwi sou andedan miray de de pous ou plis, nou rékòmandé ke ou itilizé kadna ak bawo fè ak panno plywood. Plasé panno plywood youn sou lòt pi bon pou fénèt ki konstwi sou andedan miray ki gen mwen ke de pous.

\section{Panno Plywood ak Kadna Bawo Fè}

Matéryo Ke Nap Bezwen:

- Fèy plywood ki gen $5 / 8$ pous épésè (Nou rékòmandé $3 / 4$ pous)

- Kadna ak bawo fè $3-4$ pous

- Drill

- $\quad$ Si sikilè

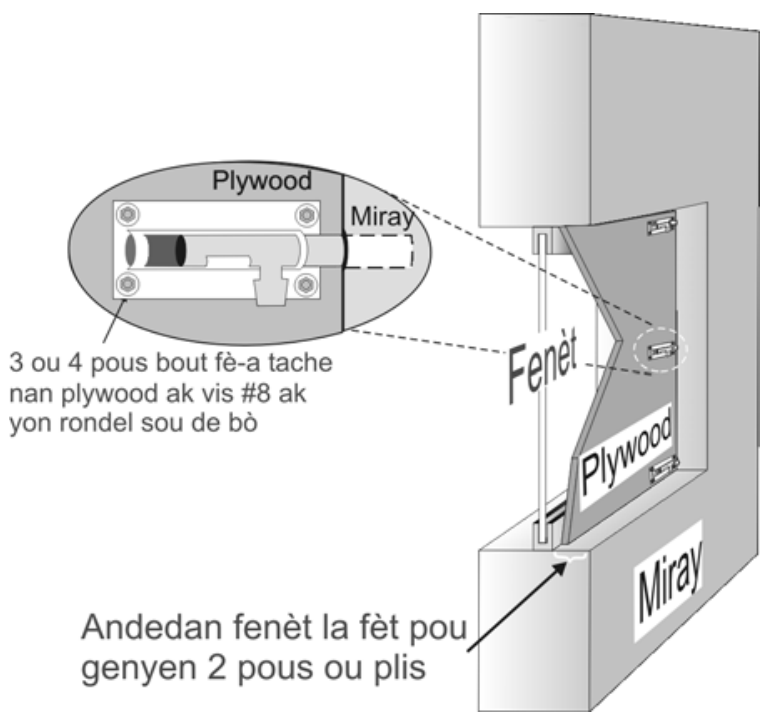

\section{Senp Diréksyon Pou Enstalasyon:}

Koupé fèy plywood-la pou li kouvri andedan fénètla. Konekté plizyè fèy ak $2 \times 4$ ouswa bon jan gon pou fénèt ki laj. Visé kadna ak bawo fè-a nan chak fèy plywood, pandan ke ou ap itilizé yon vis pou chak 12 pous nan sans vètikal fèy-la. Maké ki bò twou-yo pral tonbé nan miray-la. Maké chak fèy avek "Anwo" ak "Anba" é ekri numewo fénèt-la. Fouyé twou é kouvri twou-yo lè ou pap itilizé-yo.

1. Dokiman sa-a fè pati 3 de 5 nan Edikasyon + Aksyon =_Koleksyon Kontwol Dega Van. Dat piblikasyon: Novanm 2005.

2. Hal S. Knowles, III, Kòdinatè, Pwogram pou Resous Bon Jan Kominoté; Kathleen C. Ruppert, Syantis Estansyon Asosyé, Sévis Estansyon Enèji Laflorid; Karla A. Lenfesty, Espesyalis Kontwol Dega Van Siklòn, Estansyon Kopératif pou Konté St Lucie, Enstiti pou manjé ak Syans Agrikiltirèl, Inivvèsiti Laflorid; Barbara Haldeman, Asistan Editè, Sèvis Estansyon Enéji Laflorid.

Enstiti pou manje ak Syans Agrikiltirèl (IMSA) se yon Opòtinité pou Anplwa Egal - Anplwayè Aksyon Afimatif gen dwa pou founi rechèch, enfòmasyon edikasyonèl ak lòt sèvis sèlman bay endividyèl ak enstitisyon kap fonksyoné san fè atansyon a ras, kwayans, koulè, relijyon, laj, enfimité, sex, oryantasyon sexsyel, stati marital, orijin nasyonal, opinyon ou afilyasyon politik. Pou enfòmasyon sou kouman pou jwenn lòt piblikasyon estansyon, pran kontak ak Biro Sèvis Estansyon Koperatif kin an Konté pa-ou. Sèvis Estansyon Koperatif Laflorid / Enstiti pou manjé ak Syans Agrikiltirèl / Inivèsité Laflorid / Larry R. Arrington, Dirèktè.

Pwogram Kado nan Depatéman Laflorid sou Afè Kontwol Konstriksyon Rezidans nan Kominoté-a pèyè yon pati nan piblikasyon-an. 


\section{Plasé Panno Plywood Youn Sou Lòt}

Matéryo Ke Nap Bezwen:

- Fèy plywood ki gen 5/8 pous épésè (Nou rékòmandé $3 / 4$ pous)

- Klou (pa buy twòp sékirité ouswa vis/boulon (buy plis sékirité) pa mwens ke 2 pous longè

- Bouchon ki fèt an plon ouswa fè di (PA itilizé plastic)

- Mato (pou klou) é ouswa drill (pou vis)

- $\quad$ Ti mòso bwa plat (Si se nésésè)

- Si sikilè

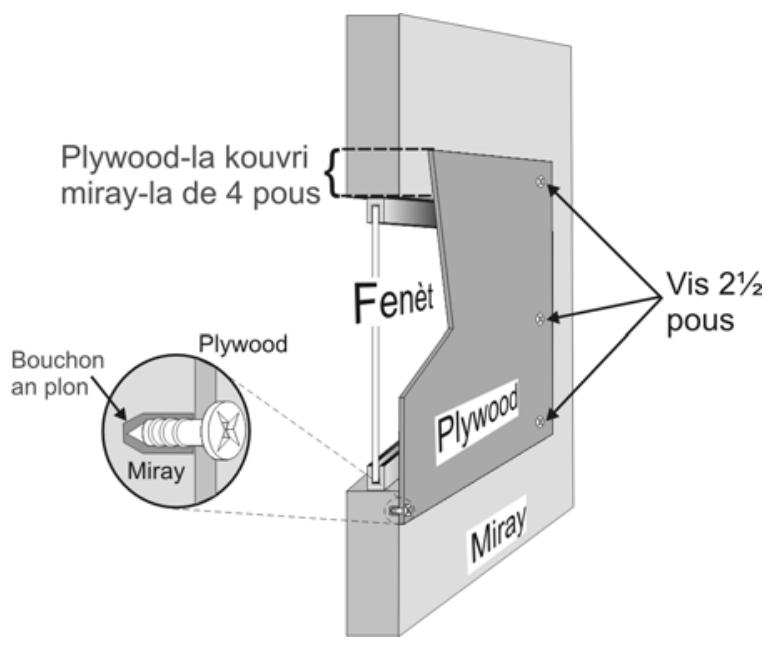

Senp Diréksyon Pou Enstalasyon:

Koupé plywood-yo pou yo kapab kouvri chak fénèt ak 4 pous enplis. Konékte plizyè fèy ak $2 \times 4$ ouswa bon jan gon pou gwo fénèt. Fouyé twou nan fèy plywood-la ak miray-la. Maké chak fèy avek “Anwo" ak "Anba” é ekri numewo fénèt-la. Rantré bouchon nan miray-la ak youn mato. Visé fèy plywood-la nan bouchon an. Bouché twou yo lè ou pap itilizé yo.

\section{Lòt Bagay Pou Nou Konsidéré}

Pa Mété FBK: Li pran 30\% plis fèy bwa konprésé pou li genyen menm fòs ak fèy plywood. Nou rékòmandé ke nou itilizé fèy plywood.

Planifyé Anvan Nou Kòmansé: Fèy plywood vann bon maché, fasil pou nou itilizé, é ou kapab jwenn li nan tout magazen ki vann matéryo. Sepandan, achté fèy plywood nou anvan siklòn nan vini. Si nou tann two lontan nou kapab pa jwenn ankò.
Séré Byen: Fèy plywood fèt pou byen sere pou nou pa domajé li. Imidite ak chalè Laflorid gen dwa défòme é gate plywood-la ki ap kòz ke nou pap kapab itilizé li. Sé youn trè bon bagay pou nou tréte plywood la kont dlo ak pwodwi éspesyal ouswa penti anvan ke nou sere li nan yon koin ki fré é sèk. Kenbé tout vis, boulon, nan youn mamit ki maké avek panno-yo. Lè nape sere fèy plywood yo mété yo kouché plat pou yo pa défòmé. Pa janmè séré fèy plywood lè yo mouyé.

Véyé Tèmit: Yon lòt bagay pou nou fè, se jwenn yon jan pou nou pa méte fèy plywood yo atè. Sa va anpéche tèmit manjé yo.

Maké Panno-yo: Pifò kay genyen diferan dimansyon fenèt. Li trè enpòtan pou nou maké chak panno pou nou kapab konnen nan ki fenèt ke panno yo pralé é ki bò panno-a pralé anwo ouswa anba. Yon lòt bon bagay pou nou konsidére se ékri sou yon mòso papyé tout nimewo fenèt-yo ak nimewo panno-yo pou nou kapab konnen ki bò pou nou méte yo.

Pou plis détay sou afè fèy panno plywood, gadé nan APA (Asosyasyon Bwa pou Konstriksyon) Wèb sayt (gadé anba-a). Kliké sou paj "Publications" é chèché pou "hurricane shutters."

\section{Lòt Resous}

APA - Asosyasyon Bwa pou Konstriksyon www.apawood.org

NOAA \& AOML | Panno Plywood pou Siklòn http://www.aoml.noaa.gov/hrd/shutters/index2.html 